\title{
Food will be our First Medicine
}

\section{Simon Brigitte*}

Department Sport and Nutrition, France

*Corresponding Author: Simon Brigitte, Department Sport and Nutrition, France.

Received: July 03, 2019; Published: July 25, 2019

DOI: $10.31080 /$ ASNH.2019.03.0382

\section{"Food will be our first medicine" Hypocrates said}

By making the organization more involved, the athlete represents a population with increased nutritional needs. However, the majority of nutritional advice in the world of sport focuses on the energy aspect (carbohydrates, proteins and lipids) while obscuring the fundamental role of micronutrients. The intensive practice of a sport tests our metabolism and demands the immune system. Infectious diseases or various inflammations are therefore common after a sports effort. This is why athletes are prone to injury and often get sick after competitions.

To prevent the body from reacting to disease, it is necessary to take care of the metabolism on the one hand by replacing the best industrially processed foods with foods rich in vitamins and minerals, and on the other hand by providing supplements. micronutrients.

The need for micronutrients skyrockets during sports

Metabolic changes associated with sports practice lead to an increase in the need for micronutrients. Physiologically, the increased supply of oxygen increases oxidative stress and the formation of free radicals.

Second, more intense metabolic activity requires a greater intake of micronutrients, while their loss through perspiration, urine and intestines increases. It is not uncommon to see athletes suffer from deficiencies in these precious substances.

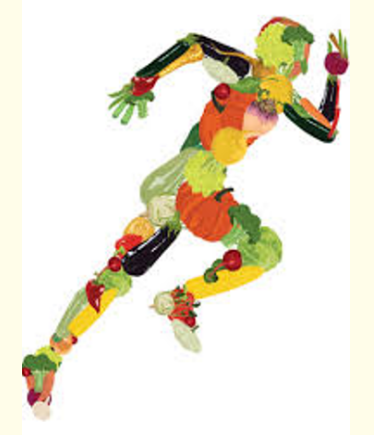

Figure 1
The importance of micronutrition for athletes

A micronutrient-rich preparation can overcome any deficiencies and neutralize increased oxidative stress, as well as the additional production of free radicals. Thanks to this approach, the athlete optimizes his performance, reduces the risk of illness and accidents and shortens his recovery period.

B vitamins support the decomposition of carbohydrates and fats. They can also act as support during fatigue, lack of initiative and reduced physical and brain performance capacity. In addition, they increase the ability to coordinate and concentrate, and prevent the drop in performance. Vitamin B1 deficiency manifests as calf cramps and muscle weakness.

Magnesium is necessary for the transformation of vitamin B1 into its active form, a magnesium deficiency leads to a deficiency in vitamin B1.

Zinc compensates for losses from sweat, strengthens the immune system and stimulates muscle processes and wound healing.

Calcium and magnesium are important for muscle function. It is therefore recommended to include magnesium-rich foods in daily food, such as wholemeal bread, wheat bran and legumes.

Iron is involved in the transport of oxygen as well as in several energy-gaining reactions in muscle cells.

A lack of iron is enough to limit performance in endurance sports.

Antioxidants, such as certain enzymes, vitamins A, C and E, zinc, manganese, selenium and beta-carotenes, can neutralize free radicals. These are trained in greater quantities during the practice of sport and can harm the cells of the body.

\section{The amateur sportsman as concerned as the pro}

As you will understand, whether one is an amateur athlete or a professional, micronutrients are very important for the transposition of energy in the metabolic process. 
Supplementing it offers several benefits: increased performance capacity, faster regeneration and recovery time (after training or competition), protection from injury and disease, rehabilitation faster after illness or injury.

\section{How do you feed your brain well?}

When we think nutrition, we too often forget what it takes tomacro-nutrientsmicronutrients to our brains. As we talk more and more about the importance of mental preparation, I think it is essential to know your brain in order to eat better. What we eat affects not only our health, our physical ability to exercise but also our cognitive abilities.

Neuronutrition at the service of our performance is the scientific approach to discover precisely how our diet affects neurons and their connections.

\section{Good fats for our neurons}

Contrary to popular belief, we need to consume fat: cholesterol, omega 3 and other fatty acids that make up all brain cells, from neurons to blood vessels. When we know that the brain uses 20\% of the body's total energy when it weighs only $2 \%$ of body weight but burns $120 \mathrm{~g}$ of pure glucose, it is essential to give it what it needs according to our physical activity.

\section{Sugars for brain energy and concentration}

Since the brain has no energy reserves in the form of sugars, it must be constantly supplied by the blood that delivers immediately usable sugars. Blood glucose (concentration of sugars circulating in the blood) is therefore crucial to our athletic performance. To achieve stable blood sugar levels during exercise, you simply need to absorb enough sugars before, during and after exercise.

\section{Oxygen supply is also important!!!}

Our brain is the organ of the human body that consumes the most oxygen: $40 \%$ of the body's oxygen is used by brain cells because it is impossible to burn glucose without it. That's why it's so important to know how to breathe well!

Omega 3: a must for our brain!
In addition to the natural anti-inflammatory effect produced by omega 3, they are truly our motor oil. They are particularly found in cold sea fish such as mackerel, tuna, herring or salmon. Unfortunately, fish are increasingly contaminated by heavy metals that pollute the seas and our daily intakes are insufficient. More and more scientists are advocating o3 supplementation.

\section{Did you know that? Vitamins: a vital factor}

Vitamins help maintain vital balance because it is through them that our cells can use the nutrients that are energy-making. Without them, carbohydrates, fats and proteins would be unusable. Each vitamin has specific functions and none can replace another. In the body, many reactions require the presence of several vitamins and the absence of one of them can interfere with the functioning of others.

Hydration: a performance parameter too often overlooked

It is as important to eat well as to hydrate. As blood irrigation and nutrient supply decrease, signs of fatigue and headaches appear. Studies have shown a decrease in short-term abilities: slower reactions, impaired lucidity, decreased concentration abilities.

You will understand: no mental health without a good diet and hydration adapted to physical exertion. These are the two essential fuels for sporting performance. But eating is not just about eating. It's also about enjoying food and sharing pleasant moments: a dimension that matters especially to the brain because that's where the key to pleasure lies.

I wish you all the best victories in the world.

\section{Volume 3 Issue 8 August 2019}

(C) All rights are reserved by Simon Brigitte.

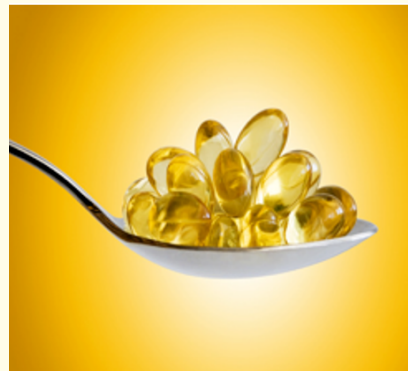

Figure 2 